\title{
SPALART-ALLMARAS TURBULENCE MODEL VALIDATION FOR AIR FLOW AROUND SKYSCRAPER MODEL
}

\author{
Ibrahim A. M. Gad ${ }^{+}$ \\ Faculty of Engineering, Zagazig University, Egypt.
}

\begin{abstract}
:
This work presents an experimental-numerical investigation of the flow around tall building model. Measured data of static surface pressure are compared with the numerical results. The main objective of the comparison was to obtain information on how well the numerical simulations using standard turbulence models, were able to reproduce the experimental data. The numerical predictions for pressure distribution on the building are performed by solving the Reynoldsaveraged Navier-Stokes (RANS) equations using both the Spalart-Allmaras (SA) and standard k- $\varepsilon$ (SKE) turbulence models constructed in FLUENT CFD code. It is known from literature that LES models are more suitable to simulate the behavior of bluff body in a crossed flow with better accuracy but from an engineering point of view they are high demanding in terms of time and CPU performances. This paper is to test the faster set of SA and SKE turbulence models and to judge their accuracy by using experimental data for validation. The results show that SA model reproduces the experimental data better than SKE. Generally SA model shows reasonable agreement with the corresponding wind tunnel pressure coefficients in different wind directions.
\end{abstract}

KEYWORDS: Aerodynamic forces, CFD, High-rise building, Turbulence models, FLUENT Code

\section{ÉTUDE EXPERIMENTALE-NUMERIQUE DES CARACTERISTIQUES AERODYNAMIQUES DU FLUX D'AIR AUTOUR DU MODELE SKY SCRAPER}

\section{RÉSUMÉ :}

Ce travail présente une étude expérimentale-numérique de l'écoulement autour du modèle grand bâtiment. Les données mesurées de la pression statique de surface sont comparées avec les résultats d'un modèle numérique. L'objectif principal de la comparaison a été d'obtenir des informations sur la façon dont les simulations numériques en utilisant des modèles de turbulence standard, ont été en mesure de reproduire les données expérimentales. Les prédictions numériques de distribution de pression sur le bâtiment sont effectuées par la résolution du Reynolds moyennées de Navier-Stokes (RANS) en utilisant les équations de la Spalart-Allmaras et modèle standard k- $\varepsilon$ turbulence (SKE) construit dans FLUENT Code. Il est connu que les modèles à partir des ERP sont plus appropriés pour simuler le comportement du corps perturbateur dans un écoulement croisé avec une meilleure précision, mais d'un point de vue technique, ils sont à forte demande en termes de performances et de temps CPU. Le présent document est de tester l'installation plus rapide des modèles de turbulence SA et SKE et de juger de leur précision en utilisant des données expérimentales pour la validation. Les résultats montrent que SA reproduirait les données expérimentales mieux que SKE. Généralement SA indique un accord raisonnable avec les coefficients de pression du vent correspondant tunnel dans différentes directions du vent.

MOTS CLES: Forces aérodynamiques, CFD, immeuble de grande hauteur, les modèles de turbulence, FLUENT Code

* Received: 5/12/2010, accepted: 24/1/2011

+ Contact author (iamg_51@yahoo.com) 


\section{INTRODUCTION}

Tall buildings structure must be able to withstand the external loads imposed by nature. Wind is one of the major forces responsible for the catastrophic failure and loss of life. The wind flow around tall building involves separation, reattachment and recirculation, which underlines the importance of unsteady simulation. Due to turbulent fluctuations of wind the buildings structures may be subjected to resonant wind loads. Accurate evaluation and prediction of wind loads are very important in reducing the adverse effects of wind in the building. Roshko (1954), Lyn et al. (1995), Williamson (1996), Luo et al. (2003) and Irwin (2008) performed pioneering work on irregular vortex shedding and turbulent properties behind bluff bodies. Flow past a square or a rectangular cross-section building have been made by many researchers (Ref. [3, 8 and 12]). Various type of turbulence models are tested for Simulation of turbulent flow over surface mounted obstacles with sharp edges and corners (Ref. [1, 2, 4, 7, 15 18]). Many turbulence models are available by CFD codes. It is a good and easy opportunity to test these available models in the CFD codes by the pressure measurements in wind tunnel. Basora and Younis (1992) have previously compared standard $\mathrm{k}-\varepsilon$ model results with wind tunnel based 2D back-step tests undertaken by Kim et al (1980). The numerical treatments by FLUENT code are based on the solution of the Navier Stokes equations, the continuity equation and the turbulence model equations (Ref. [13 and 19]).
Lubcke et al (2001) noticed that the large eddy simulation model (LES) is able to simulate the behavior of bluff body in a crossed flow with good accuracy. But from an engineering point of view the LES model demands a long working time and high CPU performances.

The goal of this search is to test the faster set of RANS turbulence models and to judge their accuracy by using experimental data for validation. Among the available turbulence models in FLUENT code, the present work considered the Spalart-Allmaras and the standard k- $\varepsilon$ turbulence models to be tested for the prediction of pressure distribution around high rise buildings. Spalart-Allmaras is a low-cost RANS model solving a transport equation for a modified eddy viscosity. This model is still relatively new and no claim is made regarding its applicability to all types of complex engineering flows. The Standard $\mathrm{k}-\varepsilon$ (SKE) model is the most widely-used engineering turbulence model for industrial applications.

\section{WIND TUNNEL TEST}

The aim of experimental test is to validate the turbulence model. The present wind tunnel is of open circuit type. A straightener built of thin sheet metal is placed at the entrance of the test section. The air mass flow rate is controlled by a gate located inside the air supply duct. The blocking ratio is nearly $4.5 \%$. Some inflow velocities profiles are measured at different mass flow rate by the use of Pitot-static tube. The building model (BM) cross section is a square of length $5 \mathrm{~cm}$ fabricated from Perspex Fig 1. 
The pressure is measured at 16 pressure taps located at the BM surface Fig 1-a by an electronic manometer which is calibrated by water manometer.

The test section is constructed to adjust the flow 2-D and to allow the vision (Fig 1-b). The BM is fixed and supported to four disks. The two parallel faces of the test section, BM, and the four disks supporting BM are fabricated from Perspex. The group composed of $\mathrm{BM}$ and the four disks is supported to the two faces of the test section. This method of support allows the BM to rotate about its axis normally to the air flow to be attacked by variable air incidence angle.

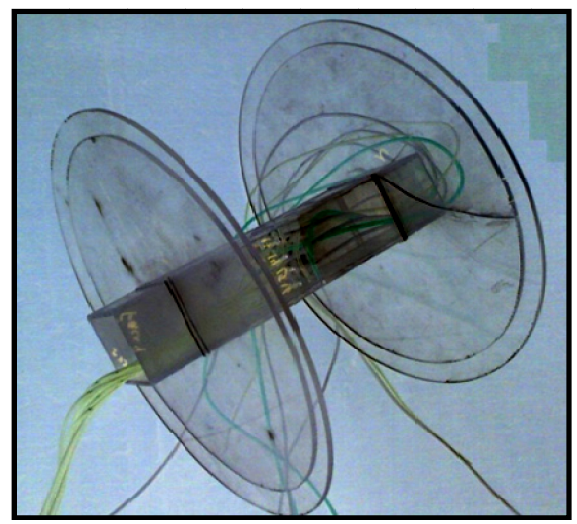

Fig. (1-a): Wind tunnel BM

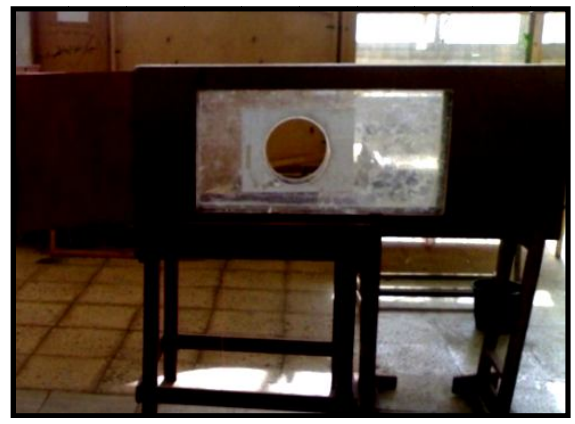

Fig. (1-b): Test section prepared for BM rotation face 2

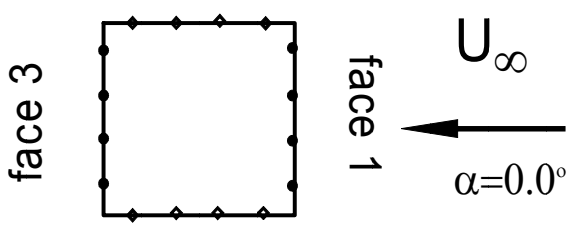

Face 4
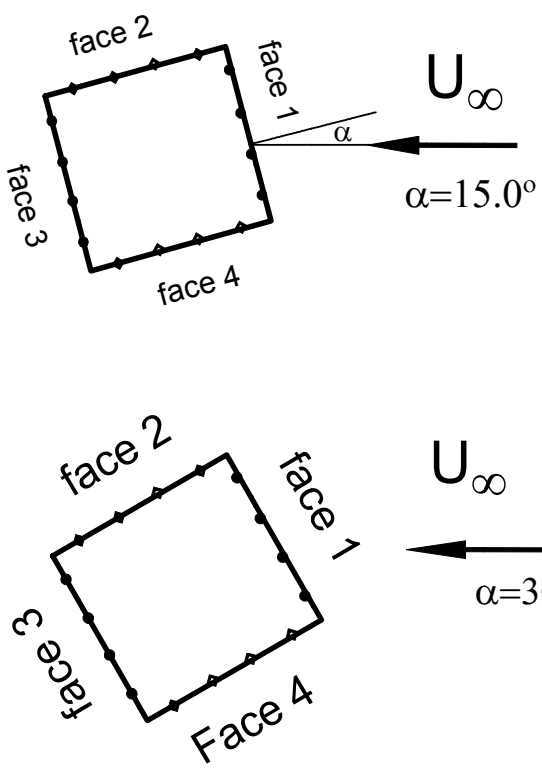

$\alpha=30^{\circ}$

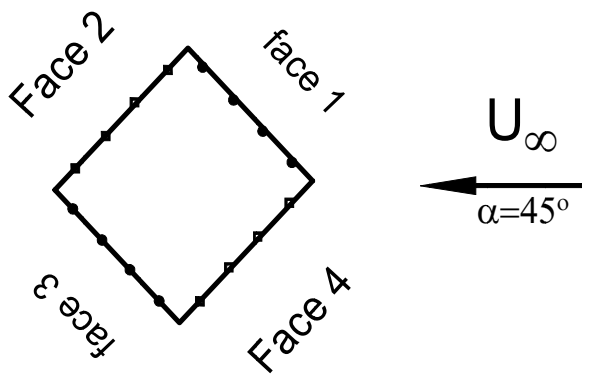

Fig. (2): Pressure taps and 4 tested wind directions; $\alpha=0.0^{\circ}, 15.0^{\circ}, 30.0$ ${ }^{\circ}$ and $45.0^{\circ}$ 


\section{NUMERICAL TREATMENT}

The numerical treatments by FLUENT code are based on the solution of Navier Stokes, continuity and turbulence model equations. Two turbulence models; Spalart-Allmaras (SA) and Standard k- $\varepsilon$ (SKE) are used here by FLUENT code to simulate the flow around skyscraper model. The flow equations are solved by applying the semi-implicit method for pressure linked equations (SIMPLE) with accuracy of the second order for all variables.

\section{1.Spalart-Allmaras Model (SA)}

SA is a low-cost RANS model solving a transport equation for a modified eddy viscosity. It embodies a relatively new class of one-equation models where it is not necessary to calculate a length scale related to the local shear layer thickness. It is designed specifically for aerospace applications involving wall-bounded flows. It has been shown to give good results for boundary layers subjected to adverse pressure gradients. This model is still relatively new. No claim is made regarding its applicability to all types of complex engineering flows. In FLUENT, however, the Spalart-Allmaras model has been implemented to use wall functions when the mesh resolution is not sufficiently fine. This might make it the best choice for relatively crude simulations on coarse meshes where accurate turbulent flow computations are not critical. Furthermore, the nearwall gradients of the transported variable in the model are much smaller than the gradients of the transported variables in the $\mathrm{k}-\varepsilon$ or $\mathrm{k}-\omega$ models. This might make the model less sensitive to numerical error when nonlayered meshes are used near walls. See Fluent guide for further discussion of numerical error. The transported variable in the Spalart-Allmaras model, $\tilde{v}$, is identical to the turbulent kinematic viscosity except in the nearwall (viscous-affected) region. The transport equation for $\tilde{v}$ is:

$\frac{\partial x}{\partial t}(\rho \tilde{v})+\frac{\partial}{\partial x_{i}}\left(\rho \tilde{v} u_{i}\right)=G_{v}+$

$\frac{1}{\sigma_{\widetilde{v}}}\left[\frac{\partial}{\partial x_{j}}\left\{(\mu+\rho \widetilde{v}) \frac{\partial \widetilde{v}}{\partial x_{j}}\right\}+\right.$

$\left.C_{b 2} \rho\left(\frac{\partial \widetilde{v}}{\partial x_{j}}\right)^{2}\right]-Y_{v}+S_{\widetilde{v}}$

$G_{v}$ is the production of turbulent viscosity and $Y_{v}$ is the destruction of turbulent viscosity that occurs in the near-wall region due to wall blocking and viscous damping. $\sigma_{\widetilde{v}}$ and $C_{b 2}$ are constants and $v$ is the molecular kinematic viscosity. $S_{\widetilde{v}}$ is a userdefined source term see FLUENT help. The model constants:

$$
C_{b 1}, C_{b 2}, \sigma_{\widetilde{v}}, C_{v 1}, C_{\omega 1}, C_{\omega 2}, C_{\omega 3}
$$

and $k$ have the following default values:

$$
\begin{gathered}
C_{b 1}=0.1355, C_{b 2}=0.622, \sigma_{\widetilde{v}} \\
=\frac{2}{3}, C_{v 1}=7.1 \\
C_{\omega 1}=\frac{C_{b 1}}{\kappa^{2}}+\frac{\left(1+C_{b 2}\right)}{\sigma_{\widetilde{v}}}, C_{\omega 2} \\
=0.3, C_{\omega 3}=2.0, \kappa \\
=0.4187
\end{gathered}
$$




\section{2. Standard $k-\varepsilon$ model (SKE)}

The most widely-used engineering turbulence model for industrial applications should be robust and reasonably accurate, contains submodels for buoyancy combustion compressibility etc. Limitations the $\varepsilon$ equation contains a term which cannot be calculated at the wall and therefore, wall functions must be used. Generally it performs poorly for flows with strong separation, large streamline curvature, and large pressure gradient.

The $k-\varepsilon$ model uses an equation for turbulence kinetic energy $(k)$ to determine the velocity scale and another equation for dissipation rate $(\varepsilon)$ to determine its length scale. In the derivation of the $k-\varepsilon$ model, it is assuming that the flow is fully turbulent, and the effects of molecular viscosity are negligible. The modeled transport Equation for $\mathrm{k}$ is written as:

$\rho \partial_{i} k U_{i}=\partial_{j}\left[\left(\mu+\frac{v_{t}}{\sigma_{k}}\right) \partial_{j} k\right]+\mathrm{G}_{\mathrm{k}}-\rho \varepsilon$ Similarly the dissipation rate $\varepsilon$ is given by the following Eq.

$\rho \partial_{i} \varepsilon U_{i}=\partial_{j}\left[\left(\mu+\frac{v_{t}}{\sigma_{\varepsilon}}\right) \partial_{j} \varepsilon+\right.$

$\left.\mathrm{C}_{1 \varepsilon} \frac{\varepsilon}{\mathrm{k}} \mathrm{G}_{\mathrm{k}}-\mathrm{C}_{2 \varepsilon} \rho \frac{\varepsilon^{2}}{\mathrm{k}}\right]$

$\mu_{t}=C_{\mu} \rho \frac{k^{2}}{\varepsilon}, \quad G_{k}=\overline{-\rho u_{i}^{\prime} u_{j}^{\prime}} \partial_{i}\left(U_{j}\right)$

The coefficients in the k- $\varepsilon$ equations which are:

$C_{\mu}=0.09, \quad \sigma_{k}=1.0 \quad, \quad \sigma_{\varepsilon}=1.3$, $C_{\varepsilon 1}=1.44$ and $C_{\varepsilon 2}=1.92$

\section{RESULTS AND DISCUSSIONS}

Some inflow velocities profiles are measured at different mass flow rate by the use of Pitot-static tube and the results are recorded in Fig. (3),
Reynolds number ranges between 37149 and 69069.

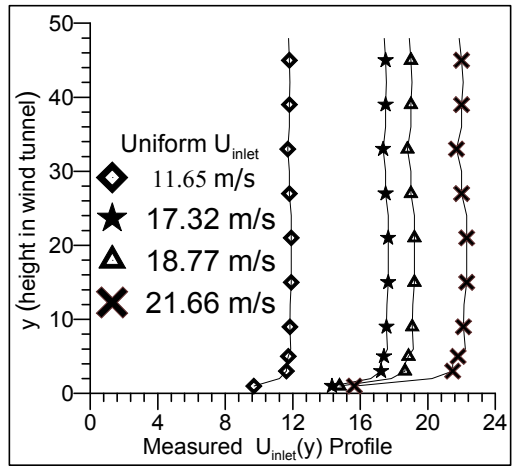

Fig. (3): Measured inlet velocity profiles $U_{i n}(y)$

\section{1. Boundary Conditions}

A schematization of the 3-D computational domain is shown in Fig 4. It is a wide parallelogram and the $\mathrm{BM}$ is located inside it normal to the flow. BM is a square prism of height $h$ and $b$ is the length of its base.

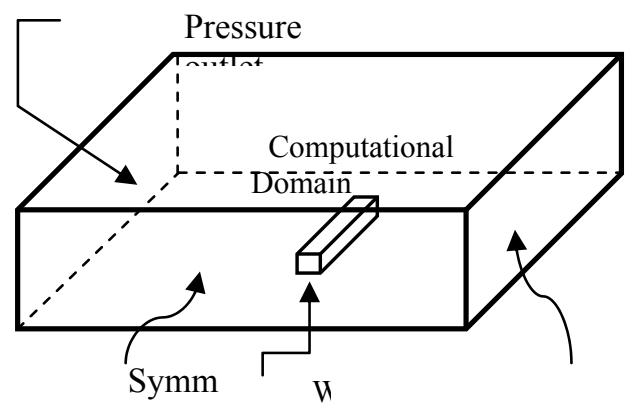

Fig. (4): Sketch of 3-D computational domain

The numerical studies are conducted in 2$\mathrm{D}$ and 3-D by SA and SKE and the predicted results are compared in between and with the experimental 2-D results. The boundary conditions are; BM wall, in-flow velocity at inlet, outlet pressure and symmetry conditions are used for the lateral borders, where it is assumed that the normal gradients for all flow variables are zero. 


\section{2. Mesh Sensitivity Analysis}

The work here aims to obtain the mean pressure distribution over the building model surface at the mid plane $\mathrm{z}=\mathrm{h} / 2$. The numbers of total gridcells required for independent solution were investigated in 2-D and 3-D for SA and SKE. Figs. (5-a) and (5-b) show 2$\mathrm{D}$ and 3-D grid, respectively. The search for grid independent solutions needs the beginning by the aid of literature. Results for numerous trials using SA model are shown in Fig 6 for 3-D flow.

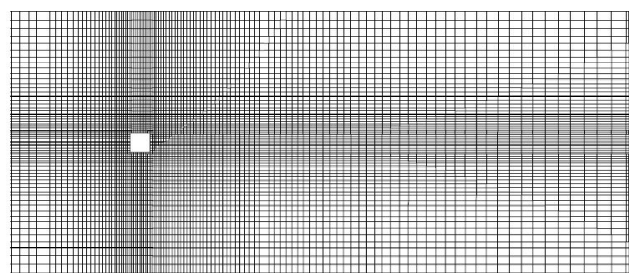

Fig. (5-a): cross section of structured grid at mid height of BM for 3-D computational domain

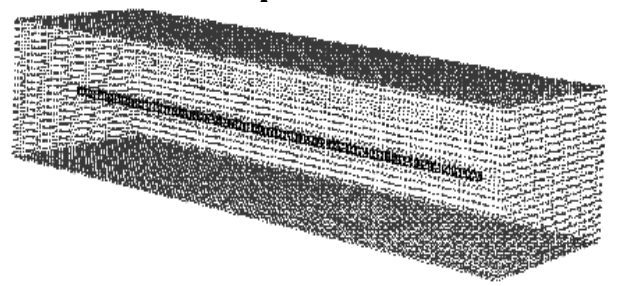

Fig. (5-b): 3-D grid structure

\section{3. Time Variant Calculation}

The time variant lift, drag and moment coefficients are investigated here by SA and SKE turbulence models. The time variant lift, drag, and moment coefficients predicted by SA are presented in Figs (7-a), Fig. (7-b) and Fig. (7-c) respectively, for 3-D flow.

\section{4. Time Averaged Calculations}

The time averaged; lift, drag, moment, base pressure coefficient and Strouhal number are calculated numerically and experimentally in 2-D and compared in Figs (8-a) through Fig (8-e).

\subsection{Flow Pattern Predictions}

The velocity magnitude field predicted by SA using FLUENT CFD Code at the interior region of computational domain for different instants $t$ is presented in Fig 9-a. The figure shows that the positive vortex clusters formed near lower edge and the negative vortex clusters formed near upper edge each clusters are shed periodically. These phenomena may be seemed as a "periodic equilibrium" at which each a cluster stops formation itself under the effect of its image vortices inside the solid body when reaches a limited strength the opposite cluster begins to form itself. Fig. (9-b) presents the velocity field at interior computational domain at different incidence angles, $\alpha=15^{\circ}, 30^{\circ}$ and $45^{\circ}$ by SKE. The figure shows that periodic equilibrium phenomena are predicted by both SA and SKE turbulence models. Fig 10 shows some smoke steam line vizualized photos around square cylinder at different icidence angle captured by Gad (2000). SA, SKE and 2-D visualized flow are agree in producing Karman street wake due to the periodic vortex shedding. 


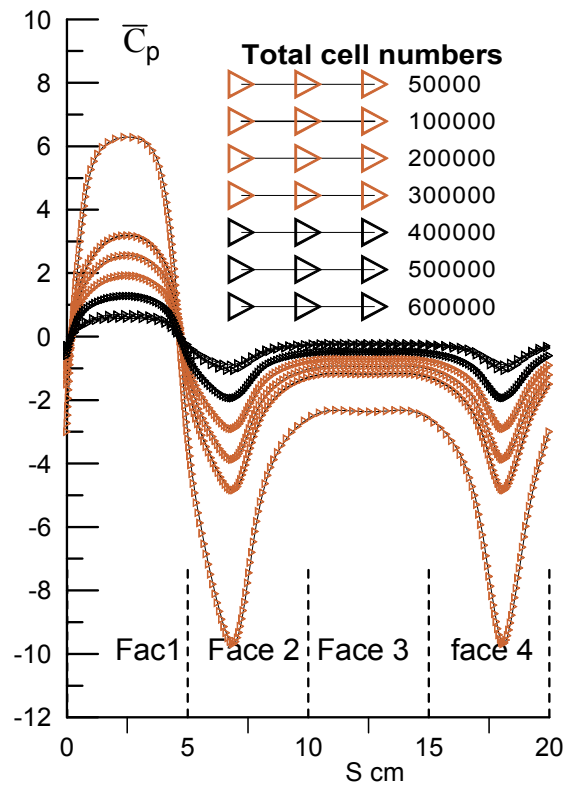

Fig. (6): Trial steps for searching the 3-D Grid independent solution $\left(\bar{C}_{p}\right)$ by $\mathbf{S A}$

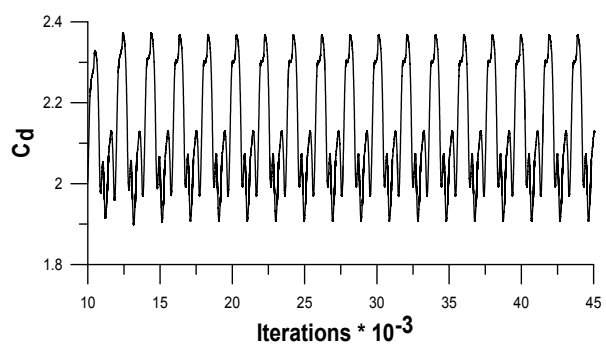

Fig. (7-a): time variant drag coefficient by $S A$

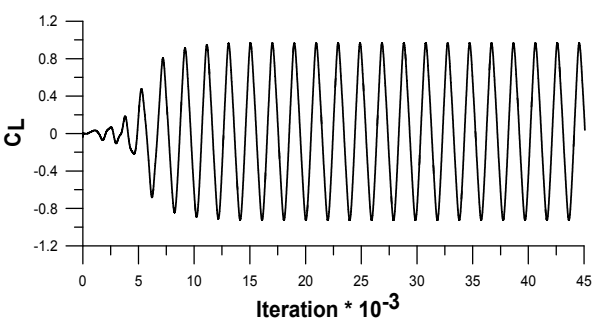

Fig. (7-b): Time variant lift coefficient by $S A$

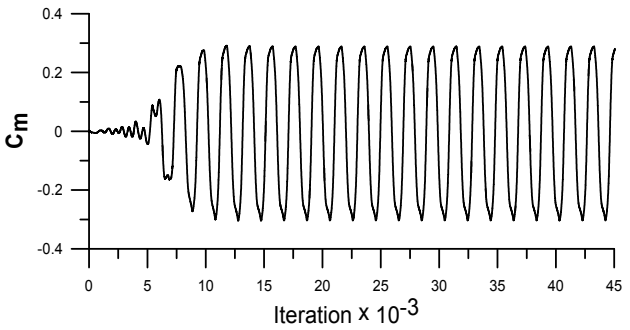

Fig. (7-c): Moment coefficient history by SA

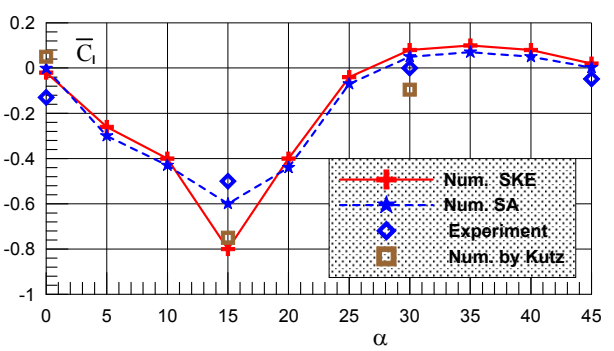

Fig. (8-a) : Lift coefficient versus incidence angle $\alpha$

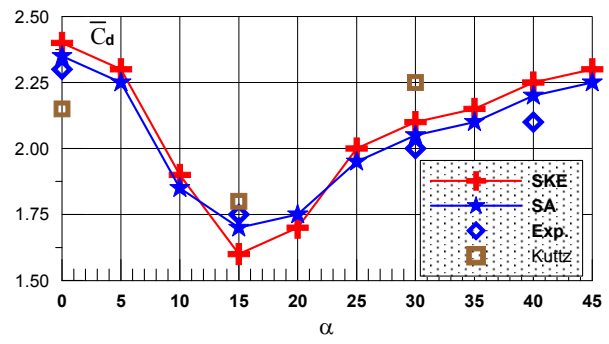

Fig. (8-b) : Drag coefficient versus incidence angle $\alpha$

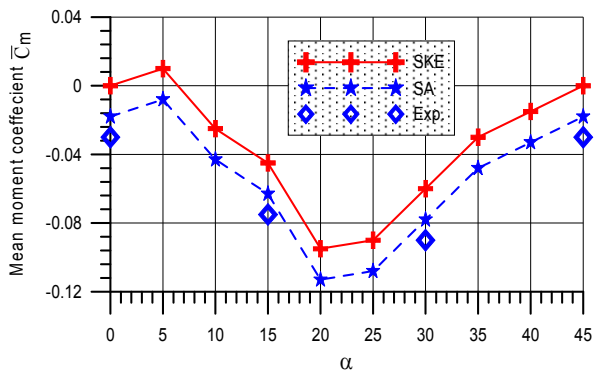

Fig. (8-c) : Moment coefficient versus incidence angle $\alpha$ 


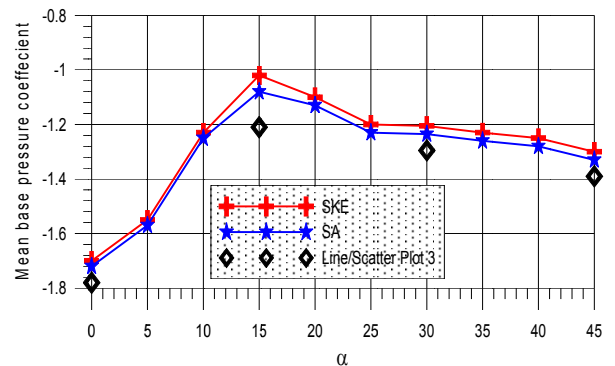

Fig. (8-d) : base pressure coefficient versus $\alpha$

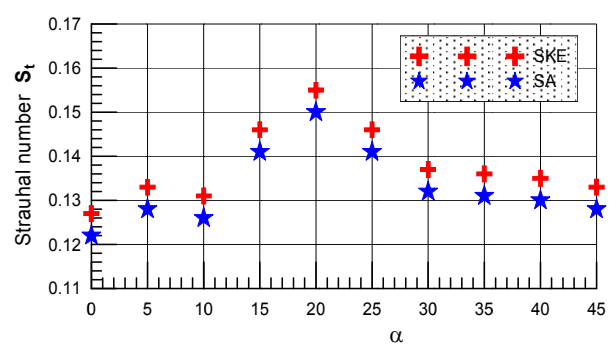

Fig. (8-e): Strouhal number versus incidence $\alpha$

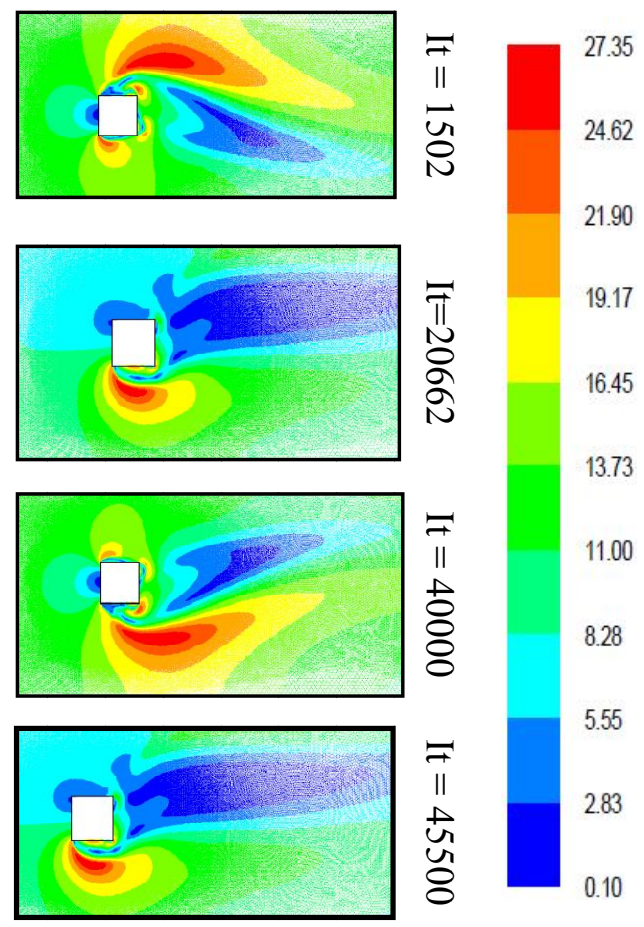

Fig. (9-a): velocity magnitude at different instants by SA (12 it $=1 \mathrm{sec})$

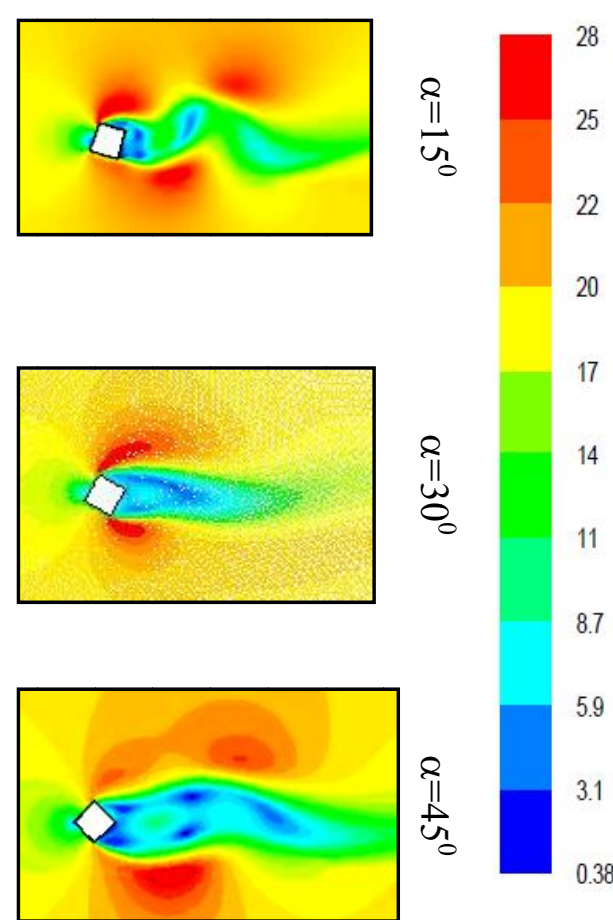

Fig. (9-b): velocity magnitude at different incidence angle by $\mathrm{SA}$
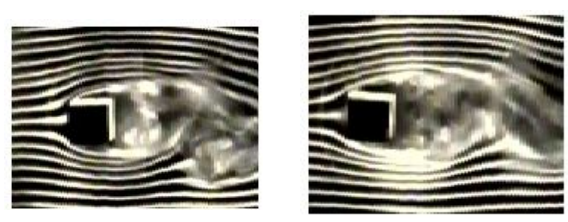

$$
\alpha=0^{\circ}
$$

$$
\alpha=0^{\circ}
$$

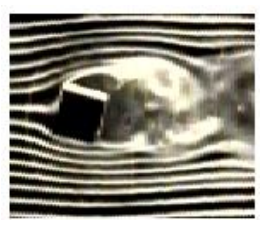

$$
\alpha=15^{\circ}
$$$$
\alpha=45^{\circ}
$$

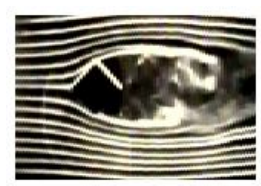

Fig. (10): Stream lines around square cylinder visualized by Gad (2000) 


\section{6. Complexity of the turbulence model}

The complexity of different turbulence models may vary strongly depending on the details one wants to observe and investigate by carrying out such numerical simulations. Complexity is due to the nature of Navier-Stokes equation ( $\mathrm{N}-\mathrm{S}$ equation). $\mathrm{N}-\mathrm{S}$ equation is inherently nonlinear, time-dependent, three-dimensional partial differential equation (PDE).

Turbulence could be thought of as instability of laminar flow that occurs at high Reynolds numbers (Re). Such instabilities originate from interactions between non-linear inertial terms and viscous terms in N-S equation. These interactions are rotational, fully timedependent and fully three-dimensional. Rotational and three-dimensional interactions are mutually connected via vortex stretching. Vortex stretching is not possible in 2-D spaces. That is also why no satisfactory 2-D approximations for turbulent phenomena are available.

Another feature of a turbulent flow is that vortex structures move along the flow. Their lifetime is usually very long. Hence certain turbulent quantities cannot be specified as local. This simply means that upstream history of the flow is also of great importance.

\section{7. Two and three dimensional study}

In this study the skyscraper model has constant square cross section in the whole height $h$ and the ratio of $B M$ height to the square length, $h / b \gg>1$, large enough and this may allows the calculation to limit the CFD study to a 2-D analysis. Fig. (11) presents mean drag coefficient predicted using SA in 2-D and 3-D computational domain for different values of $\mathrm{b} / \mathrm{h}$. This figure shows that it may be reasonable in this study to make the comparison of the 2D experimental results with the numerical results in 2-D or 3-D at mid cross-section $\mathrm{h} / 2$ of a skyscraper model which have a large height relative to its base length. Therefore the numerical studies are conducted in 2-D and 3-D by SA, SKE and the 2-D tunnel test.

Figs (12 to 15) are the results of three dimensional computational domains and the two dimensional test in wind tunnel. The aim is the comparison between experimental and predicted pressure coefficients distributed around the skyscraper model cross-section in the mid plane at different incidence angle by SA and SKE turbulence models. The experimental pressure distribution presented in these figures generally show reasonable agreement with experimental tunnel testing, except at the corners where separation and high pressure gradient occur.

The figures show that the obtained results are in good agreement with the experimental data when using the Spalart-Allmaras model; smaller local differences in pressure suctions are mainly noted at points of separation. The Standard k- $\varepsilon$ shows larger local differences at points of separation, larger than those obtained by SpalartAllmaras model. 


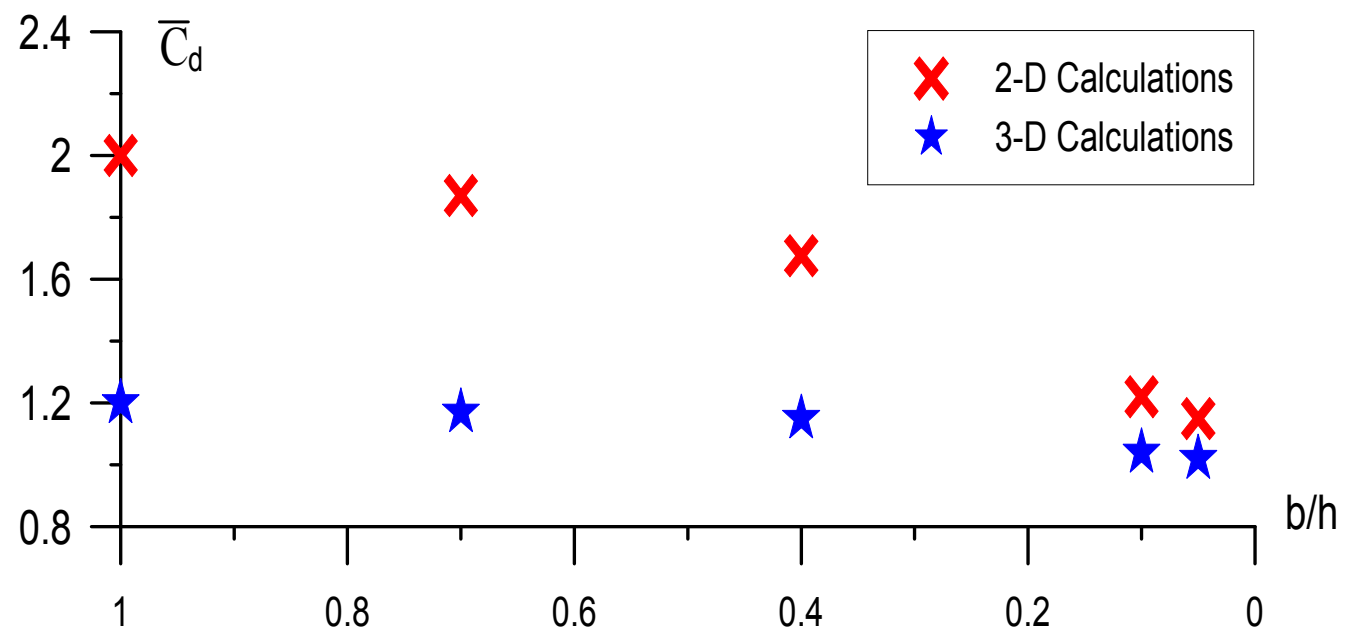

Fig. (11): 2-D and 3-D mean drag coefficient for different b/h by SA

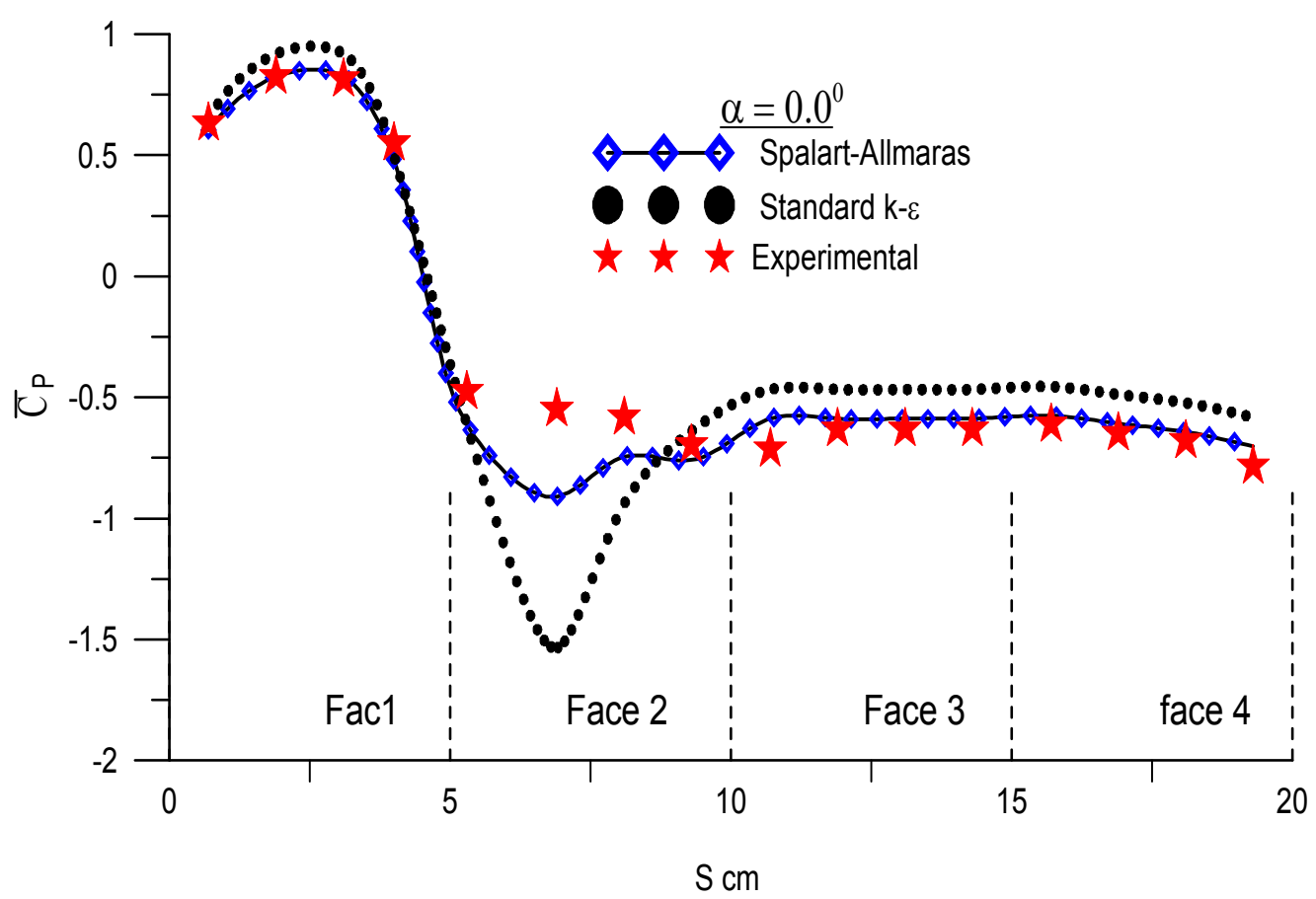

Fig. (12): Comparison between experimental and predicted $\overline{\mathbf{c}}_{\mathrm{P}}$ by $\mathrm{SA}$ and SKE distributed around the skyscraper model cross-section in the mid plane $\alpha=\mathbf{0 . 0 0}$ 


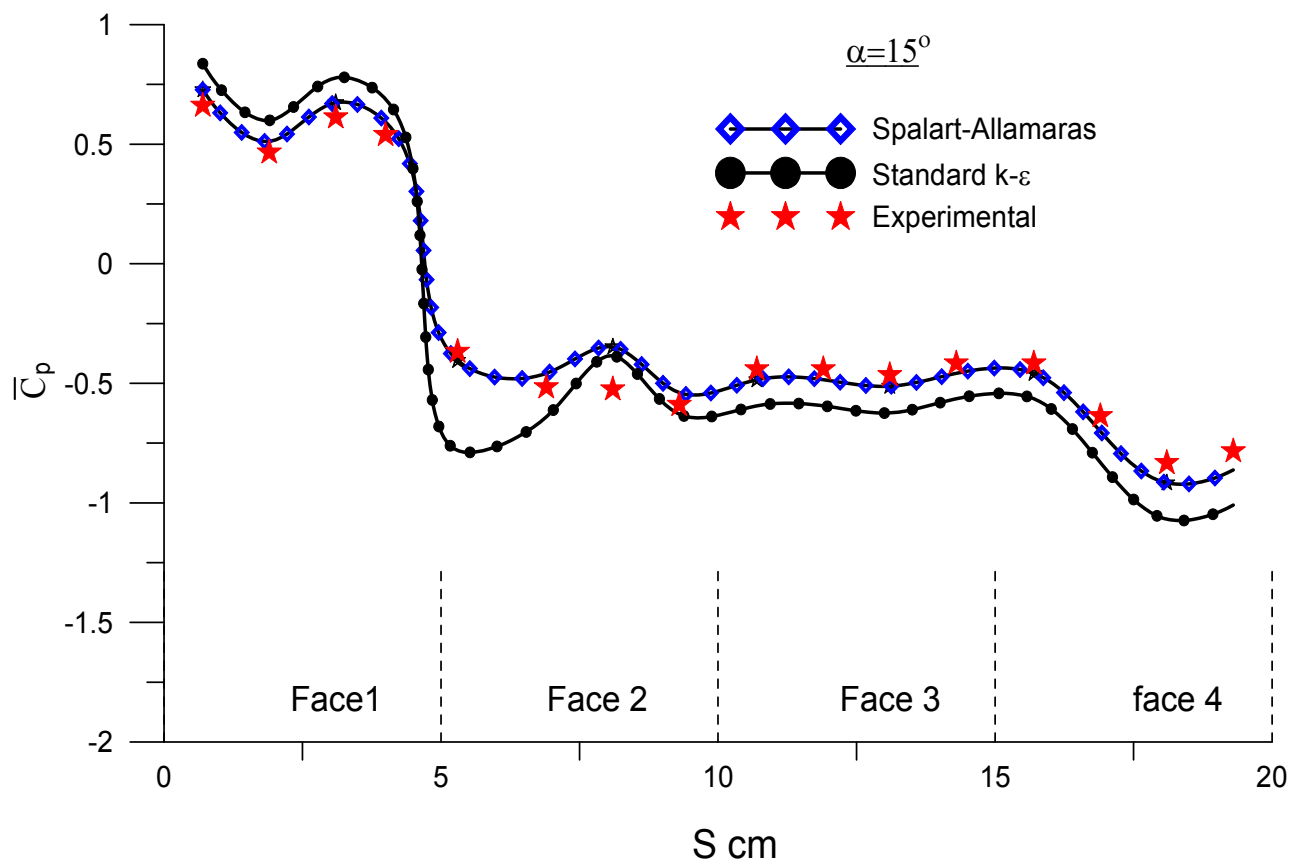

Fig. (13): Comparison between experimental and predicted $\bar{c}_{P}$ by SA and SKE distributed around the skyscraper model cross-section in the mid plane $\alpha=15^{\circ}$

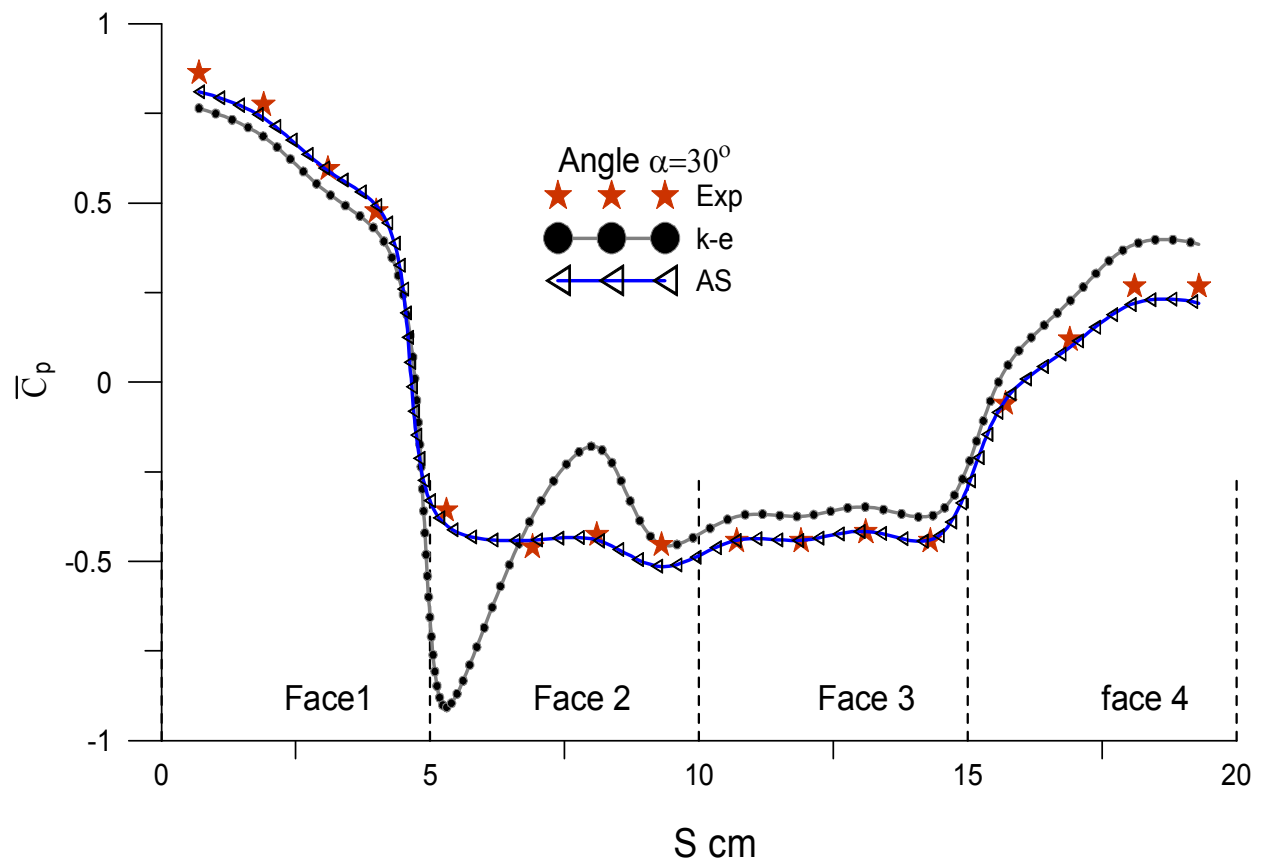

Fig. (14): Comparison between experimental and predicted $\bar{c}_{P}$ by SA and SKE distributed around the skyscraper model cross-section in the mid plane $\alpha=3^{\circ}$ 
Spalart-Allmaras Turbulence Model Validation For Air Flow Around Skyscraper Model Ibrahim A. M. Gad

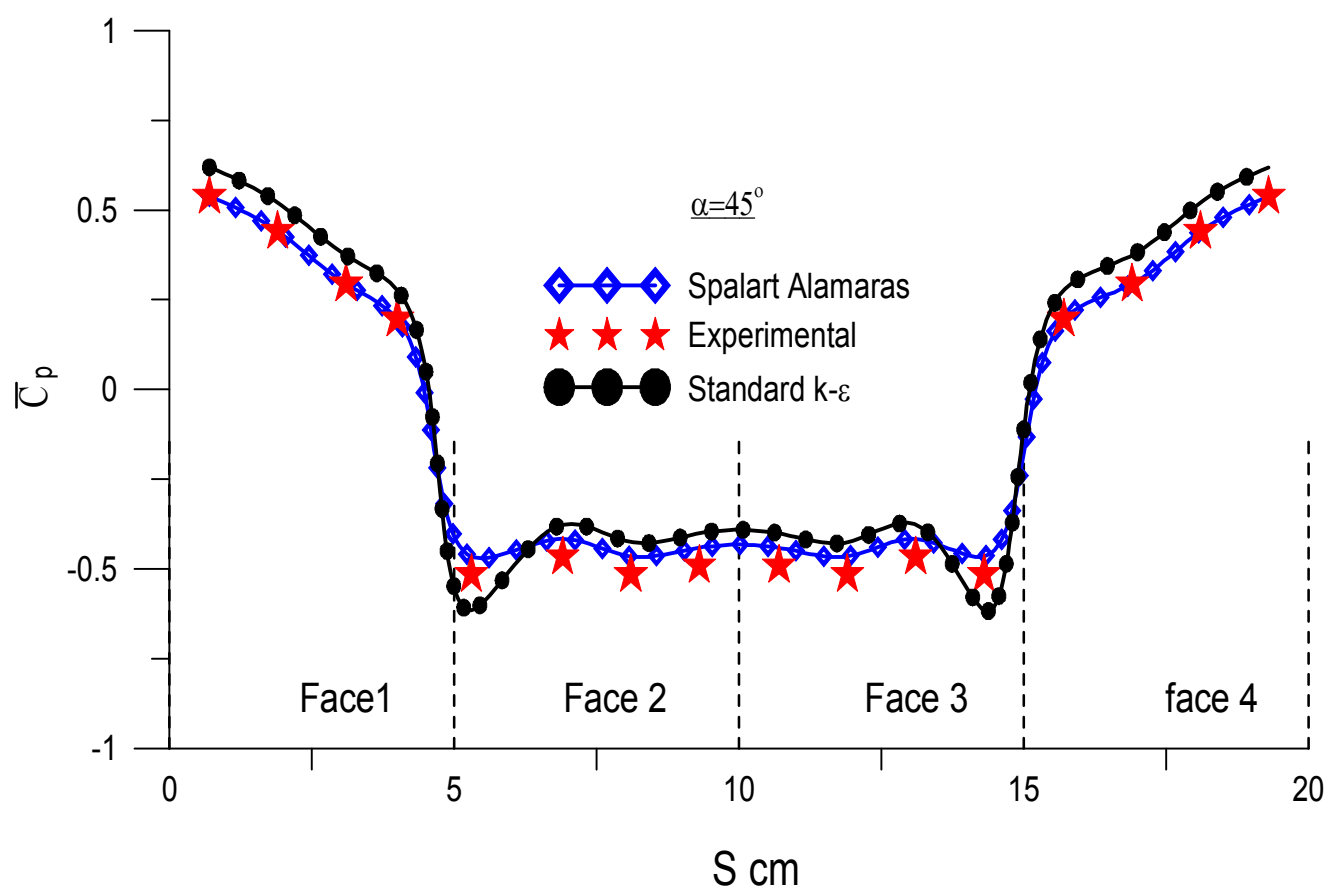

Fig. (15): Comparison between experimental and predicted $\bar{c}_{P}$ by SA and SKE distributed around the skyscraper model cross-section in the mid plane $\alpha=45^{\circ}$ 


\section{CONCLUSION}

The Spalart-Allmaras and the Standard k- $\varepsilon$ turbulence models are used in this investigation to calculate the distribution of pressure coefficient around a tall building model. The FLUENT code is used to conduct the calculations. The results obtained by simulation model were found in good agreement with the experimental data when using the Spalart-Allmaras model; local differences in pressure suctions are mainly noted at points of separation, smaller than that obtained experimentally.

The Standard k- $\varepsilon$ simulation showed somewhat reasonable agreement with experimental results; local differences in pressure suctions are mainly noted at points of separation, larger than those obtained from wind tunnel measurements. It can be noted that the Standard k- $\varepsilon$ approach demanded larger computational time than SpalartAllmaras. The standard k- $\varepsilon$ approach is more sensitive meshing processes and needs more computational efforts for evaluation of the grid independent solution.

Therefore, it can be concluded that the Spalart-Allmaras model gives a better overall performance, providing a reasonable prediction in terms of global response and capturing the physics of the flow with minor computational effort.

\section{REFERENCES}

1- Baetke, F., Werner, H., (1990), "Numerical simulation of turbulent flow over surface mounted obstacles with sharp edges and corners", J. Wind Eng. Indust. Aerodyn. 35 pp 129-147.

2- Basora, B., Younis, B., (1992), "Progress in the prediction of turbulent wind loading on buildings", J. Wind Eng. Indust. Aerodyn., 41-44 pp 2863-2874.

3- Courchesne, J., Laneville, A., (1982), "An experimental evaluation of drag coefficient for rectangular cylinders exposed to grid turbulence", Trans. ASME: J. Fluids Eng. 104, 523-527.

4- Craft, T., Launder, B. E., Suga, K., (1993), "Development and application of a cubic eddyviscosity model of turbulence", Int. J. Heat Fluid flow 17 pp 108115.

5- Gad, I. A., (2000), "Refined Model of Discrete Vortex Method Applied to Sharp Edged Bodies", Journal of Engineering and Applied Sciences, Faculty of Engineering, Cairo University, Vol. 48-3.

6- Irwin, P.A., (2008), "Bluff body aerodynamics in wind engineering", J. of Wind Eng. Ind. Aerodyn. 96, pp 701-712. 
7- Kim, J., Kline, S., Johnston, J., (1980), "Investigation of a reattaching turbulent flow over a backward facing step", Trans. ASME 102 pp 302-308.

8- Kwok, K. C. S., (1983), "Effects of turbulence on the pressure distribution around a square cylinder and possibility of reduction", Trans. ASME: J. Fluids Eng. 105, 140-145.

9- Lubcke, H., Scmidt, St., T. Rung, F. Thiele, (2001), "Comparison of LES and RANS in bluff-body", Journal of Wind Engineering and Industrial Aerodynamics.

10- Luo, S.C., Chew, Y. T., Ng, Y. T., (2003), "Characteristics of square cylinder wake transition flows" Phys. Fluids 15, pp 2549-2559.

11- Lyn, D. A., Einav, S., Rodi, W., Park, J. H., (1995), "A laserDoppler velocity study of the ensemble-averaged characteristics of the turbulent near wake of a square cylinder", J. Fluid Mech. 304, pp 285-319.

12- Okajima, A., (1982), "Strouhal number of rectangular cylinders", J. Fluid Mech. 123, pp 379-398.

13- Pope, S.B., (2000), "Turbulent flows", Cambridge University, Cambridge.

14- Roshko, A., (1954), "On the development of turbulent wakes from vortex streets", NACA Report 1191.

15- Spalart, P. R., and Allmaras, S. R., (1991), "A One-Equation Turbulence Model for Aerodynamic Flows," AIAA 920439.

16- Speziale, C., (1987), “On nonlinear $\mathrm{k}-\mathrm{l}$ and $\mathrm{k}$-(models of turbulence)", J. Fluid Mech. 178 pp $459-475$.

17- Speziale, C., Ngo, T., (1988), "Numerical solution of turbulent flow past a backward facing step using a nonlinear k-e model", Int. J. Eng. Sci. 26 pp 1099-1112.

18- Suga, K., (1996), "Development and Application of a Non-linear Eddy Viscosity Model Sensitized to Stress and Strain Invariants", Ph.D., Department of Mechanical Engineering, UMIST, Manchester.

19- Wilcox, D.C, (1998), "Turbulence Modelling in CFD", DCW Industries.

20-Williamson, C. H. K., (1996), "Vortex dynamics in the cylinder wake", Ann. Rev. Fluids Mech., vol. 28, pp 477-539. 